\title{
Detection of n-dimensional shape deformities using n-dimensional quasi-conformal maps
}

\author{
Hei Long Chan and LoK Ming Lui
}

\begin{abstract}
Detecting deformities on objects is a typical topic in shape analysis, and has much applications such as abnormalities detection in medical imaging (e.g. growth of tumor, spread of cancer). While many algorithms are already well-established in a 2-dimensional case when the object is indeed a surface, a model that still performs well in the general n-dimensional case is still missing. It is our goal in this paper to complete this missing piece, by introducing an indicator in order to effectively distinguish between normal and abnormal deformities. The proposed framework is closely related to the classic 2-dimensional conformal geometry and quasiconformal geometry. In this work, we model abnormal deformations by anisotropic deformations. Given any two objects of the same dimension (with landmark constraints in between), we define the Anisotropic Indicator, a locally defined real-valued function on the original object, which demonstrates the abnormalities in the deformation between them. Both global and local features about the abnormalities between the two objects can be tracked by analyzing the indicator. We tested the algorithm by detecting deformations on synthetic data and real data, and results show that our algorithm can detect deformations of different types and degrees.
\end{abstract}

\section{Introduction}

In shape analysis, detection of deformities is of great importance with applications in many aspects especially in medical research. For example, in pulmonary imaging, doctors examine patients based on detecting abnormalities from the X-ray photos of the respiratory system. In cardiac imaging and oncology, to monitor disease progression and evaluate treatment effectiveness, physicians keep track on shape deformations in organs. Therefore, developing an effective method to detect abnormalities and track abnormal geometric changes over time is always in great demand.

In this paper, we model abnormal deformations by anisotropic deformations, and a framework for detecting abnormal changes on any n-dimensional 
objects via conformal geometry is to be proposed. In the first place, we will apply an existing algorithm to register the two given n-dimensional objects. After that, we will propose an indicator, which is closely related to the classic 2-dimensional quasi-conformal geometry, to quantify implicit abnormalities in the deformations between them. Lastly, after further post-processing, both global and local features about the deformation can be studied easily and any hidden abnormalities can be extracted effectively.

During investigation of medical images, normal geometric deformations in human organs can never be simply modelled as isometry. For example, stretching and contraction of lungs during normal respiration are nonisometric changes. Enlargement of human organs due to aging is also nonisometric but is regarded as normal in real life. However, we note that local geometry of organs are well-preserved in those normal situations, whereas not preserved in abnormal situation such as development of cancer and growth of tumor. Because conformal maps are well known to preserve local geometry of a surface, this motivates us to measure abnormal deformities by relating them to conformality distortion. But the notion of conformal geometry is restricted to 2-dimensional space only. In 2-dimensional space, we have the famous complex dilation (or equivalently, the Beltrami coefficient), which is uniquely assigned to each map, to measure how far the corresponding function is from being conformal. And the function is said to be conformal if and only if its corresponding Beltrami coefficient is zero. Hence, the Beltrami coefficient is an effective indicator measuring the degree of abnormalities, so a similar notion in higher dimensional spaces is of our interest. Eventually, this allows us to analyze the deformities between any two given objects, so that ultimately abnormal situations can be easily distinguished and detected.

The main contribution of this paper is the proposal of the Anisotropic Indicator to extract any implicit abnormal situation occurred in deformations applied to objects. Given arbitrary two objects of the same dimension, we can compare them by computing the indicator between them. Both global and local features can be further extracted from the indicator, such that a different kind of study of the deformation can be conducted according to desired applications. Experimental results provide evidences that our algorithm is sensitive enough to deformation of an object in a different degree, plus the region of abnormal deformations can be located and visualized.

The paper is organized as follows. In section 2, prior work on closely related topics will be reviewed. Basic mathematical background composed mainly of concepts of conformal geometry in 2-dimensional space will be included in section 3 . Then, we will describe and explain, in section 4, our 
proposed model to detect abnormal deformations between n-dimensional objects by computing the conformality distortion. Numerical algorithm will be discussed in section 5 and experimental results will be demonstrated in section 6 . Conclusions and future works are presented in section 7 .

\section{Previous work}

Different approaches have been employed to detect deformities in 2-dimensional shapes. Tosun et al. [1] proposed to use shape index, curvedness and L2 norm of mean curvature to quantify cortical gyrification and complexity. Although the notion of curvature is already well defined in n-dimensional spaces, these quantities are affected by normal changes, such as growth of organ due to aging which decreases curvature. Also, since curvature involves second-order derivative, it is too sensitive to noise in data and causes much error in numerical computation. Chung et al.[2] suggested to study changes in cortical surface area, thickness and curvature on cortical surface by computing surface Jacobian and applying statistical inference via random field theory. Although surface Jacobian is less sensitive to noisy data, it is still affected by healthy changes like normal growth of tissues which increases surface area. Then, to study gyrification patterns in Williams syndrome, Shi et al. [3] proposed to measure the degree of gyrification on cortical surfaces by the number of branches in the Hamilton-Jacobi skeletons. This method gives fast measure of complexity of the cortical surface, but subtle changes could occur within a branch of the cortical surface, which require a finer measure to detect.

Much research has been done to detect disease as well as abnormalities in medical images. Liu et al. [4] proposed to use sparse representation based classification method for computer-aided cancer diagnosis. Song et al. [5] proposed to examine medical images by a classification method called Large Margin Local Estimate. By sub-categorizing the reference images, a local estimate is performed to the test images to detect possible diseases. However, those approaches are restricted to the use of dictionary or reference images and comparison between two general images cannot be directly done. While Kim et al. [6] proposed to use a multi-spectral MRI-based clinical decision support approach to carry out automated seizure focus lateralization in patients with temporal lobe epilepsy, Lui et al. [7] proposed to study deformations using Beltrami coefficients. In their work, they formulated abnormal deformities as non-conformal deformations, and detect them by computing the Beltrami coefficient associated uniquely to the quasi-conformal maps between surfaces. The advantage of Beltrami coefficient is as follows. 
Firstly, healthy deformations such as normal breathing of lungs or normal growth of organs will not be detected since all these are conformal change of surfaces. Secondly, abnormal deformities such as growth of tumor can be tracked since they are not conformal changes of surfaces. Additionally, Beltrami coefficient involves only first-order derivative so it is not too sensitive to noisy data. This motivates us to detect abnormal deformations in n-dimensional objects using the idea of conformal and quasi-conformal geometry.

Landmark-based registration is commonly used in real applications especially in medical imaging. It has been widely studied. Wang et al. [8] [9] [10] [11] proposed to obtain the optimized harmonic registrations by minimizing an energy involving the landmark-mismatching term. Although the computed harmonic maps can better aligns the landmarks, the landmarks still can not be perfectly matched and bijectivity may be lost if a large number of landmarks is employed. Tosun et al. [13] proposed to combine parametric relaxation, inverse stereographic projection and iterative closest point registration to align cortical sulci across brain surfaces. Bookstein et al. [12] proposed to obtain a registration with soft landmark constraints by applying a biharmonic regularization. However, the former two diffeomorphisms computed still cannot match landmarks perfectly. Another approach is the use of time-dependent vector fields, which is also widely studied [14] [15] [16] [17] [18]. Using time-dependent vector fields, landmarks can be matched well under an iterative process finding a diffeomorphism under the flow. The time-dependent vector fields facilitate the optimization procedure but requires more memory and the computational cost is generally expensive. Recently, Wei et al. [19] proposed to compute quasi-conformal mappings for feature matching face registration. However, exact landmark matching and bijectivity of the resultant map are not gauranteed under their proposed algorithm. Lately, Lee et al. [20] proposed an algorithm to compute the n-dimensional quasi-conformal mappings via an energy minimization. The tools are powerful since landmark constraints are exactly matched and the algorithm works well even when severe deformations are employed.

\section{Mathematical background}

In this section, basic concepts on the conformal geometry in 2-dimensional space will be reviewed to enlighten the idea of our proposed model in the next section. 


\subsection{Conformal maps}

Given two 2-dimensional manifolds $M$ and $N$, a diffeomorphism $f: M \rightarrow N$ is said to be conformal if it preserves angles between any two tangent vectors in the tangent space of a point $x \in M$. More precisely, since $f$ is a map from $M$ to $N$, the differential $d f: T M \rightarrow T N$ is a map from the tangent bundle of $M$ to the tangent bundle of $N$. By denoting $d_{x} f=$ $d f(x, \cdot)$, we define $f$ to be conformal if $d_{x} f: T_{x} M \rightarrow T_{f(x)} N$ satisfies $\left\langle d_{x} f(u), d_{x} f(v)\right\rangle=\lambda(x)\langle u, v\rangle$ for any $u, v \in T_{x} M$, for some smooth function $\lambda: M \rightarrow \mathbb{R}$. Therefore, a conformal map preserves surface metric up to a multiplicative factor called the conformal factor, which is denoted above as $\lambda$.

Another definition of conformal maps is that, a diffeomorphism $f$ is conformal if it satisfies the Cauchy-Riemann Equation:

$$
\frac{\partial f}{\partial \bar{z}}=0
$$

where $\frac{\partial}{\partial \bar{z}}=\frac{\partial}{\partial x}+i \frac{\partial}{\partial y}$. Readers may check that the above two definitions of conformal maps are equivalent. In the remainder of this paper, we will focus on the latter definition since we can generalize the notion of conformal maps to the notion of quasi-conformal maps, which is intuitively describing mappings that are "close to conformal".

\subsection{Quasi-conformal maps}

A homeomorphic mapping $f: M \rightarrow N$ is defined to be quasi-conformal if it is orientation-preserving and satisfies the Beltrami equation:

$$
\frac{\partial f}{\partial \bar{z}}=\mu(z) \frac{\partial f}{\partial z}
$$

where $\mu: M \rightarrow \mathbb{C}$ is Lebesgue measurable satisfying $\|\mu\|_{\infty}<1$, and $\frac{\partial}{\partial z}=$ $\frac{\partial}{\partial x}-i \frac{\partial}{\partial y} \cdot \mu$ is called the Beltrami coef ficient, which is a measure of nonconformality.

An obvious observation is that, $\|\mu\|=0$ if and only if $f$ is conformal. The relation between conformal map and quasi-conformal map can be made clearer by investigating the local property of them. Infinitesimally, for any point $z$ in a small neighborhood of a point $p \in M$, a quasi-conformal mapping $f$ has its local parameter expression:

$$
\begin{aligned}
f(z) & \approx f(p)+f_{z}(p) z+f_{\bar{z}}(p) \bar{z} \\
& =f(p)+f_{z}(p)(z+\mu(p) \bar{z})
\end{aligned}
$$




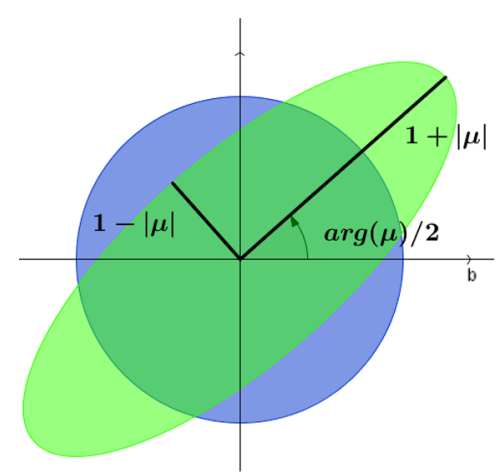

Figure 1: Illustration of the conformality distortion in 2-dimensional space: mapping an infinitesimal disk to an infinitesimal ellipse.

Since the translation function $f(p)$ and the dilation function $f_{z}(p)$ are conformal, all the non-conformality of $f$ is due to the term $D(z)=z+\mu(p) \bar{z}$, which causes $f$ to eventually map infinitesimal circle to infinitesimal ellipse (See Figure 1). Specifically, the angle of maximal magnification is $\arg (\mu(p)) / 2$ with magnifying factor $1+|\mu(p)|$ while the angle of maximal contraction is the orthogonal angle $(\arg (\mu(p))-\pi) / 2$ with contraction factor $1-|\mu(p)|$.

To conclude, there is a one-to-one correspondence between any quasiconformal mapping $f: M \rightarrow N$ and the associated Beltrami coefficient $\mu$. Therefore, the Beltrami coefficient $\mu$ characterizes the behaviour of $f$, which can be used to measure the abnormality of $f$ to the given surface in application [7]. This eventually leads to the construction of a similar measure in arbitrary dimension space, which is our task in the next section.

\section{Proposed model}

In this section, firstly, details of our proposed Anisotropic Indicator will be explained. This is to be done by examining the construction of the Beltrami coefficient in detail. The proposed indicator is able to quantify the abnormality of an n-dimensional diffeomorphism. In the next subsection, we will discuss the registration process to reconstruct the diffeomorphism between two n-dimensional objects with landmark constraints. Finally, an investigation will be done on the computation of our proposed indicator.

\subsection{Anisotropic Indicator}

In two dimensional space, the Beltrami coefficient plays the role of an indicator, measuring the behaviour of its corresponding function $f$. Concerning 
the infinitesimal ellipse in Figure 1, the ratio of the major axes to the minor axes characterizes the ellipse and hence the corresponding quasi-conformal mapping. This ratio is crucial in quantifying the degree of deformation that $f$ induces. And in high dimensional spaces, a similar ratio still exists.

Let $S$ be a high dimensional infinitesimal sphere and let $x, r$ be its center and radius respectively. A high dimensional quasi-conformal mapping $f$ maps $S$ to an infinitesimal ellipsoid $E$. For any distinct $u, v \in S, f(u)$ and $f(v)$ are in $E$. Considering the supremum of the ratio of their distance to the center of the ellipsoid $f(x)$ over $S$, this characterizes the local property of $f$. Therefore, we have the following definition:

Definition 1 (Anisotropic Indicator of a homeomorphic map). Suppose $M$ and $N$ are two $n$-dimensional subsets of $\mathbb{R}^{n}$, each being connected. Let $f$ : $M \rightarrow N$ be homeomorphic, $x \in \operatorname{int}(M)$, and $S_{x}^{M}(r)$ be an (n-1)-dimensional sphere of radius $r$ centered at $x$ in $M$. The Anisotropic Indicator of $f$ at $x$ is defined to be

$$
\operatorname{Aid}_{f}(x)=\frac{L_{f}(x)-1}{L_{f}(x)+1}
$$

where $L_{f}: M \rightarrow N$ is defined by

$$
L_{f}(x):=\lim _{r \rightarrow 0} \sup _{\substack{u, v \in S_{x}^{M}(r) \\ u \neq v}} \frac{|f(u)-f(x)|}{|f(v)-f(x)|}
$$

In the above definition, $L_{f}$ determines the local property of $f$ by considering its behaviour on an infinitesimal ball in $M$. Aid $d_{f}$ is a scaling of $L_{f}$, such that $\operatorname{Aid}_{f}(x) \in[0,1)$ for any $x \in M$, and for arbitrary n-dimensional $M$. Clearly, $\operatorname{Aid}_{f}(x)=0$ if and only if $L_{f}(x)=1$ and this is true provided that $f\left(S_{x}^{M}(r)\right)$ is a sphere, and vice versa. As $\operatorname{Aid}_{f}(x)$ is bounded above by 1 , this indicator can be used to determine if $f$ is close to be conformal at $x$ or not.

Employing the above definition, we now can define a measure of abnormality of the deformation between objects of the same dimension:

Definition 2 (Anisotropic Indicator of deformation from object $M$ to object $N)$. Suppose $M$ and $N$ are two $n$-dimensional subset of $\mathbb{R}^{n}$, each being connected. Suppose $\hat{f}: L_{M} \rightarrow L_{N}$ is the landmark constraint function where $L_{M} \in M$ and $L_{N} \in N$ are the sets of landmark constraints in $M$ and $N$ respectively, with $\left|L_{M}\right|=\left|L_{N}\right|$. If $f: M \rightarrow N$ is a diffeomorphic registration from $M$ to $N$ such that $\left.f\right|_{L_{M}}=\hat{f}$, then the Anisotropic Indicator of the 
deformation from $M$ to $N$ through $f$ at $x$ is defined to be:

$$
\operatorname{Aid}_{M, N}(f ; x):=\operatorname{Aid}_{f}(x)
$$

With this definition, one is able to quantify the degree of abnormal deformation between any objects (of the same dimension). In particular, $\operatorname{Aid}_{M, N}(x)=0$ implies the deformation is conformal, and hence is normal, at $x$. When $\operatorname{Aid}_{M, N}(x)$ grows larger, it indicates that the deformation is leaving away from being normal at $x$.

Moreover, we can quantify the general and overall degree of abnormality between objects $M$ and $N$ through $f$ by defining the following General Abnormality Indicator:

Definition 3 (General Anisotropic Indicator from object $M$ to object $N$ through $f$ ). Suppose $M$ and $N$ are two $n$-dimensional subset of $\mathbb{R}^{n}$, each being connected. Suppose $\hat{f}: L_{M} \rightarrow L_{N}$ is the landmark constraint function where $L_{M} \in M$ and $L_{N} \in N$ are the sets of landmark constraints in $M$ and $N$ respectively, with $\left|L_{M}\right|=\left|L_{N}\right|$. If $f: M \rightarrow N$ is a diffeomorphic registration from $M$ to $N$ such that $\left.f\right|_{L_{M}}=\hat{f}$, then the General Anisotropic Indicator of the deformation from $M$ to $N$ through $f$ at $x$ is defined to be:

$$
\operatorname{GAid}_{M, N}(f ; x):=\int_{M} \operatorname{Aid}_{M, N}(f ; x) d x / \int_{M} d x
$$

where $d x=d x_{1} \wedge d x_{2} \wedge \cdots \wedge d x_{n}$ is the volume form of $M$ when $M$ is considered as a Riemannian manifold.

\subsection{The registration model}

In the previous subsection, we proposed an indicator such that, given a diffeomorphic registration between two objects, we can quantify the abnormality of the deformation in between. So in this subsection, our main focus is on solving for the desired diffeomorphic registration.

Our method mainly follows the algorithm proposed in [20]. Here we explain the model briefly and readers are recommended to refer to [20] for a detailed explanation. The main idea is to minimize an energy functional to solve for the desired map. Concerning the elements of the functional, note that by computing a diffeomorphic registration, we mean to find a smooth function with one-one correspondence between the objects such that landmark constraints are all satisfied. Therefore, our energy functional to be used is:

$$
\inf _{f \in F}\|H(x)\|_{1}+\frac{\sigma}{2}\|\Delta f(x)\|_{2}^{2} d x
$$


where $\sigma \geq 0$ is a fixed parameter and $F$ is the set of functions satisfying the landmark constraints.

This energy is composed of two terms. The first term $H(x)$ is a term that generically involves $D f$, the Jacobian of $f$. This term guarantees the map to be bijective and orientation-preserving having no overlaps. And for performance consideration, we choose to minimize $\|H(x)\|_{1}$. Then the second term, which is the Laplace-Beltrami operator of $f$, is employed to promote smoothness of the resultant mapping.

\subsection{Computation and discretization of the Anisotropic Indicator}

In this subsection, the discrete model and the computational method of the proposed Anisotropic Indicator are to be introduced. For a discrete model of the registration model, readers are advised to read [20] for detailed explanation.

In a discrete case, $M$ and $N$ are high dimensional meshes. Hence, for any vertex point $p \in M$, we may take the one-ring vertices of $p$, that is, all vertices in $M$ that are connected to $p$ by an edge. In the following we denote the set of all one-ring vertices of $p$ by $R_{p}$.

We may represent $M$ and $N$ as subsets of $\mathbb{R}^{n}$ by an index matrix. For example, if $M$ is a 3 -dimensional subset of $\mathbb{R}^{3}$, we can put $M$ into an, say, 1,000-by-1,000-by-1,000 matrix $\hat{M}$, and assign 1 to $\hat{M}(x, y, z)$ if $(x, y, z)$ is in $M$ and 0 otherwise. Also, if there are two adjacent 1 in $\hat{M}$ we say these two vertices are connected and the edge length is 1 . In this manner, for each $p \in M, R_{p}$ is the set of all vertices in $M$ having distance 1 from $p$. By this simplification we have the following definition of discretized Anisotropic Indicator.

Definition 4 (Discrete Anisotropic Indicator of deformation from object $M$ to object $N$ through $f$ ). Let $f: M \rightarrow N$ be a registration function. The Anisotropic Indicator from $M$ to $N$ through $f$ at each $p \in M$ is defined to be:

$$
\operatorname{Aid}_{M, N}(f ; p)=\operatorname{Aid}_{f}(p):=\frac{L_{f}(p)-1}{L_{f}(p)+1}
$$

where

$$
L_{f}(p):=\max _{\substack{u, v \in R_{p} \\ u \neq v}} \frac{|f(u)-f(p)|}{|f(v)-f(p)|}
$$

Moreover, since now $R_{p}$ is finite, we can simplify $L_{f}(p)$ by 


$$
L_{f}(p)=\frac{\max _{u \in R_{p}}|f(u)-f(p)|}{\min _{v \in R_{p}}|f(v)-f(p)|}
$$

Therefore, the Anisotropic Indicator can be easily computed in the discrete case. And by this, the discretization of the General Anisotropic Indicator is immediate.

\section{Algorithm}

In this section, we summarize the numerical algorithm to detect abnormalities of an object from another object.

Given $M$, an n-dimensional object as a control object. Given $N$, an ndimensional object that is viewed as a deformation of $M$, such that $M$ and $N$ has the same number of points (i.e. $|M|=|N|=P$ ). Given $L_{M} \in M$, $L_{N} \in N$ and $\hat{f}: L_{M} \rightarrow L_{N}$, where the former two sets are the landmarks in $M$ and $N$ respectively such that $\left|L_{M}\right|=\left|L_{N}\right|$, and $\hat{f}$ is the bijective function matching the landmark constraints. The algorithm is as follows:

1. Compute the registration diffeomorphism $f: M \rightarrow N$ by solving the minimization problem

$$
\inf _{f \in F}\|H(x)\|_{1}+\frac{\sigma}{2}\|\Delta f(x)\|_{2}^{2} d x
$$

subjected to the constraint $\left.f\right|_{L_{M}}=\hat{f}$.

2. For each $p \in M$, compute

$$
\operatorname{Aid}_{M, N}(f ; p)=\frac{L_{f}(p)-1}{L_{f}(p)+1}
$$

where

$$
L_{f}(p)=\frac{\max _{u \in R_{p}}|f(u)-f(p)|}{\min _{v \in R_{p}}|f(v)-f(p)|}
$$

3. Compute

$$
\operatorname{GAid}_{M, N}(f ; p)=\left(\sum_{i=1}^{P} \operatorname{Aid}_{M, N}(f ; p)\right) / P
$$

to measure the general abnormalities of the deformation of $N$ from $M$.

4. Local abnormalities of $N$ from $M$ can be detected by $\operatorname{Aid}_{M, N}(f ; p)$ for each point $p \in M$. 


\section{Experimental results}

We tested our proposed model on synthetic examples to validate the sensitivity of our algorithm to detect deformations of different type, different degree and different position. We also applied our algorithm to real examples like the CT lung images. After computing the Anisotropic Indicator Aid, the GAid, the maximum and the standard deviation of Aid are further computed, that each of them carry significant information to analyze the deformation. While GAid is already explained in previous sections, $\max (A i d)$ indicates the upper bound of the degree of deformation, and $\operatorname{std}(A i d)$ gives further illustration on the distribution of the deformation. Since the conformality distortion is locally defined, the distribution of the deformation can hence be visualized, by showing the color map corresponding to the magnitude of $A i d(x)$, such that abnormal deformities can be clearly located in an efficient manner.

\subsection{Synthetic examples}

In this subsection, experiments are to be done on four synthetic examples.

The first experiment is enlargement of an unit cube. In the experiment the cube is sliced into 32-by-32-by-32 small cubes evenly. For instance, each sub-cube is a cube with length $\frac{1}{32}$. The collection of vertices of all these subcubes form the vertex set of our experiment. Then, the unit cube is enlarged into cubes with edge length:

$$
\begin{cases}\text { Experiment } 1(a) & l=2 \\ \text { Experiment } 1(b) & l=4 \\ \text { Experiment } 1(c) & l=9\end{cases}
$$

Simulating normal growth of human tissue, this deformation should be regarded as entirely normal, that is, conformal, by the algorithm. The statistical result of the conformality distortion computed is recorded in table 1 . The result generated by our algorithm is as expected, proving that conformal deformation is neglectable using our proposed algorithm.

Our second experiment is to stretch the unit cube with respect to one principal axis. For simplicity the stretching is always directed along the $\mathrm{z}$ axis. And it elongates the height $h$ of the cube from $h=1$ to:

$$
\begin{cases}\text { Experiment 2(a) } & h=1.5 \\ \text { Experiment } 2(b) & h=3 \\ \text { Experiment } 2(c) & h=5\end{cases}
$$




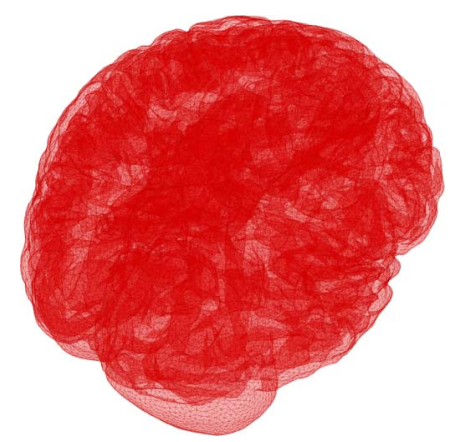

Figure 2: Volumetric brain model.

While the previous experiment models normal deformation, abnormal deformation is modelled this time. The aim performing this experiment is to show the ability of our algorithm detecting abnormal deformities. Referring to the result listed in table 1, our algorithm detected all three abnormal deformities by reporting $A i d(x)>0$ at all the points. This satisfies our expectation, meanwhile the magnitude of Aid further indicates the degree of the deformations concerned, in which it is gradually magnified as the height of the cube is further stretched.

Combining the above two experiments, the results prove the assertion that our algorithm can distinguish between normal and abnormal deformities, which are modelled by isotropic and anisotropic deformations respectively. We now proceed to investigate the sensitivity of our algorithm in the following experiments. To better model practical situations like medical imaging, we will use a volumetric brain model to perform our experiment (See Figure 2).

We first model a simpler situation: a tumor is grown inside the brain. In the experiment, we artificially plant a tumor in our brain model as indicated in Figure 3.

Now, we operate our algorithm on the brain, registering the normal one to one with an artificial tumor. We expect the algorithm to report abnormal deformation near the region of the tumor and report normal away from that region. And, fulfilling our hope, the statistical result of $\operatorname{Aid}(x)$ (in table 1) tells that abnormal deformation occurs in a localized manner, since small GAid and $s t d(A i d)$ are recorded, together with a large magnitude of $\max ($ Aid).

This is not the whole story unless the position of the deformation can also be tracked efficiently by our algorithm. Since the conformality distortion Aid is locally defined, a natural way to locate the region of abnormal 


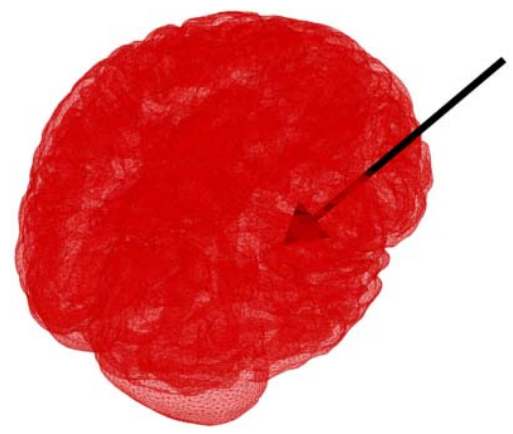

(a) position

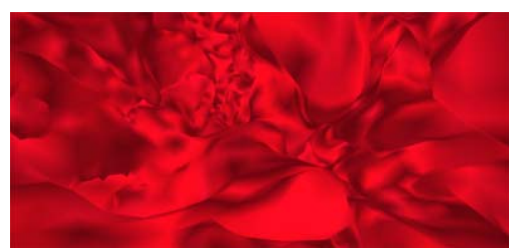

(b) Before planting

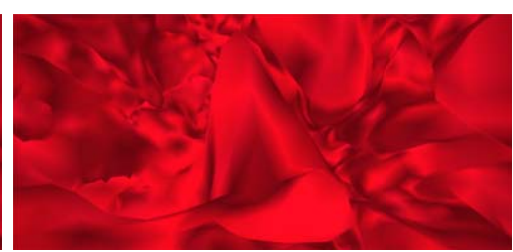

(c) After planting

Figure 3: Tumor planting in Experiment 3.

deformation is to simply search for the region where $\operatorname{Aid}(x)$ is high. But a more efficient and humane method is to use color-map with correspondence to the magnitude of $A i d(x)$. In our experiment, we colored the brain model with face color value based on the magnitude of $\operatorname{Aid}(x)$ on each face (See Figure 4).

Comparing with Figure 3, it is undoubtable that the color-map Figure 4 is capable to be used to trace the location of the tumor. Here we set the color range to be from 3 to 3.5 to ease visualization. To locate the abnormalities in a more meticulous manner, the color range can be adjusted to highlight the region with the most severe deformation. Considering the practical usage of the algorithm in industry or hospital, this should be an efficient method to locate abnormal deformations.

Our last experiment on synthetic examples is an advanced version of the last tumor experiment on brain. This time, three tumors are planted inside the brain. (See Figure 5). All the processes stated above are performed once again to detect the tumors.

The significance of this experiment is to further test the power of our algorithm. Whether the three tumors can all be identified, and how the statistical results differ from that of the previous experiment are the two most important subjects of our investigation. The results are recorded in 


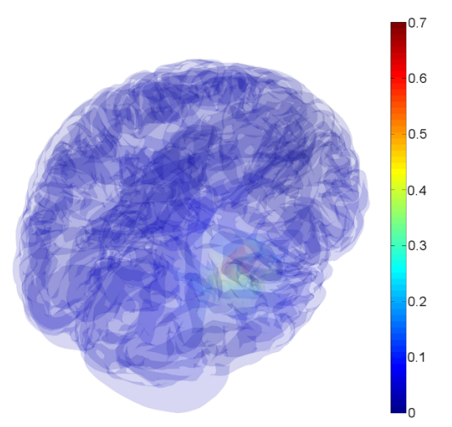

Figure 4: Color-map of brain model in Experiment 3.

table 1. Statistics show that the abnormalities occur locally somewhere. Comparing with the last experiment, $\max (A i d)$ increases slightly, which is as expected, because the tumors planted are in around the same size as that in the previous experiment. GAid remains similar, with a possible reason being that the the overall shape of the brain does not change a lot either by planting one tumor or three tumors. Lastly, std(Aid) increases significantly, which also lies on our expectation since the spread of tumors is obviously more severe this time.

Only by recording statistical results could no one understand the deformation complete enough, and the missing information is mostly stored in the color-map of Aid on the object concerned. For instance, the location of the tumors can be traced by looking at the color map.

Referring to Figure 5, the color-map is trust-worthy pointing out the tumors' location. Again, adjusting the range of the color-map varies the accuracy of location of abnormalities.

The statistical results of all four synthetic experiments are listed in the following table:

Table 1: Result of experiments on synthetic examples

\begin{tabular}{|c|c|c|c|c|c|}
\hline Deformation Type & Level & $\max ($ Aid $)$ & GAid & $\operatorname{std}($ Aid $)$ & $\max \left(\|\nabla f\|_{2}\right)$ \\
\hline \multirow{3}{*}{ Enlargement deformation } & 1(a) & 0 & 0 & 0 & 0.00146 \\
& 1(b) & 0 & 0 & 0 & 0.01172 \\
& 1(c) & 0 & 0 & 0 & 0.13348 \\
\hline \multirow{3}{*}{ Stretch deformation } & 2(a) & 0.2000 & 0.2000 & 0 & 0.00028 \\
& 2(b) & 0.5000 & 0.5000 & 0 & 0.00055 \\
& 2(c) & 0.6667 & 0.6667 & 0 & 0.00092 \\
\hline Brain tumor deformation 1 & - & 0.7386 & 0.0144 & 0.0187 & 0.00020 \\
\hline Brain tumor deformation 2 & - & 0.7553 & 0.0138 & 0.0223 & 0.00027 \\
\hline
\end{tabular}




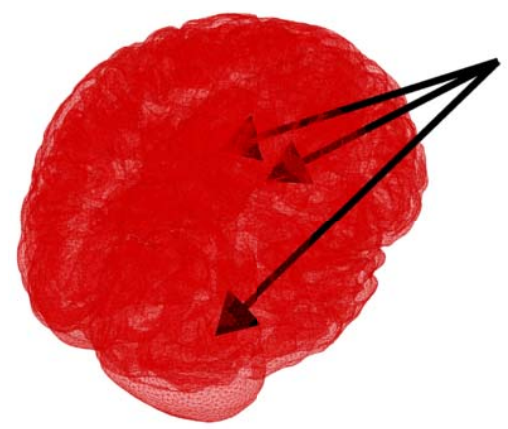

(a) position

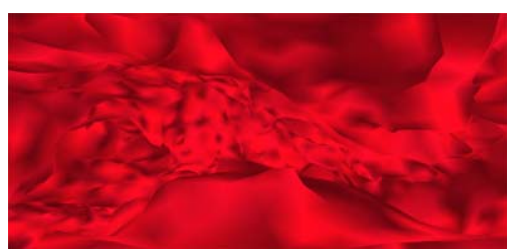

(b) tumor 1: Before planting

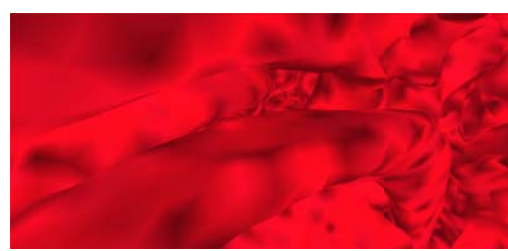

(d) tumor 2: Before planting

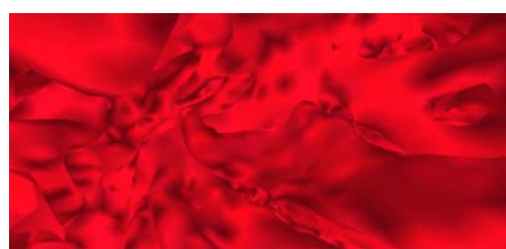

(f) tumor 3: Before planting

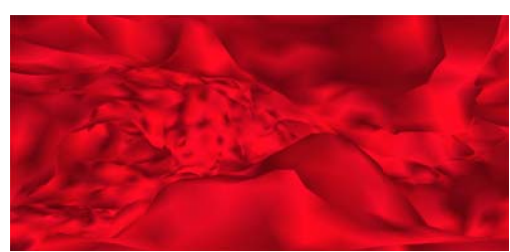

(c) tumor 1: After planting

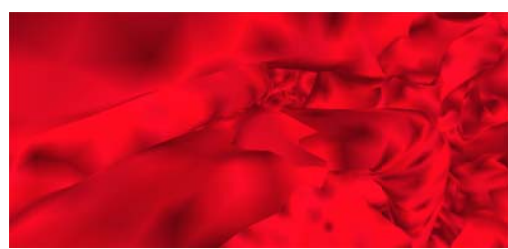

(e) tumor 2: After planting

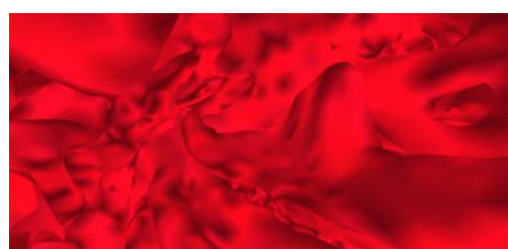

(g) tumor 3: After planting

Figure 5: Tumor planting in Experiment 4.

\subsection{Real examples using CT images of lung respiratory deformation}

Apart from synthetic examples, we also applied our algorithm to detect abnormal deformities on real data. We emphasize once again, that our algorithm is built so as to detect and extract abnormal (anisotropic) defor- 


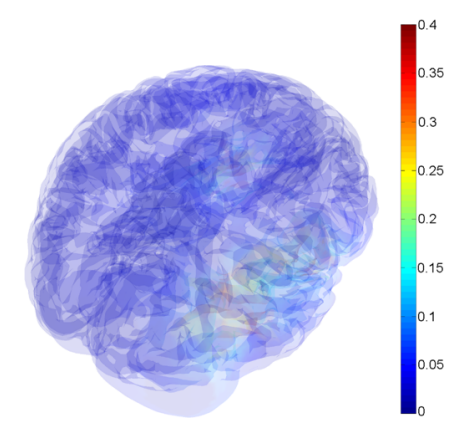

Figure 6: Color-map of brain model in Experiment 4.

mations from any hybrid deformations, hindering all normal (isotropic) deformations that may be included. Hence, in order to validate the power of our algorithm to accomplish this task, we need to analyze some hybrid deformations in real life.

For instance, we analyze the pulmonary system of human. Lungs of everyone must undergo contraction and dilation in breathing, but the way they deform may differ according to the medical state of the person. In our experiments, we used lung CT images as our data set. We tested our algorithm using five set of data. In each set of data, a sequence of lung CT images, capturing the shape of the lungs when a patient respire, are photographed. Six images, taken in constant time interval, complete a half-cycle of the respiration process, showing the shape of the lungs from maximum exhalation to maximum inhalation. Between each two consecutive images, 300 landmark constraints are pre-assumed to correspond the images. The images are partly provided (to save space) in Figure 7.

We applied our algorithm to study the five images. The statistical results are computed and recorded in table 2.

Results show that all five deformations are similar, and are very likely to be abnormal locally at some points. Among all examples, as max (Aid)

Table 2: Real example on lung CT images (note: volume of the cubes are all 1)

\begin{tabular}{|c|c|c|c|c|}
\hline Example & $\max ($ Aid $)$ & GAid & std $($ Aid $)$ & $\max \left(\|\nabla f\|_{2}\right)$ \\
\hline CT 1 & 0.6285 & 0.0424 & 0.0307 & 0.000004 \\
\hline CT 2 & 0.4658 & 0.0466 & 0.0327 & 0.000005 \\
\hline CT 3 & 0.6266 & 0.0564 & 0.0386 & 0.000006 \\
\hline CT 4 & 0.8019 & 0.0639 & 0.0401 & 0.000035 \\
\hline CT 5 & 0.6410 & 0.0490 & 0.0317 & 0.000005 \\
\hline
\end{tabular}




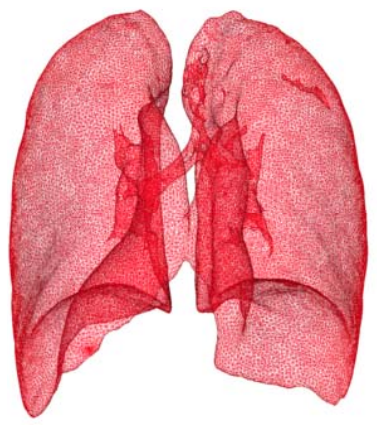

(a) Image 1 (CT1)

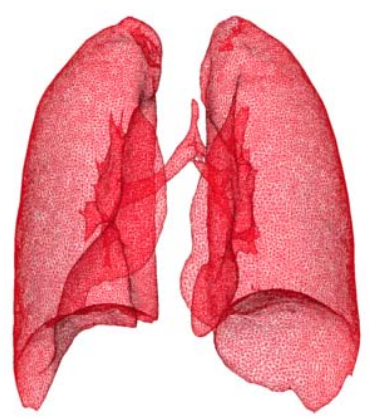

(b) Image 2 (CT2)

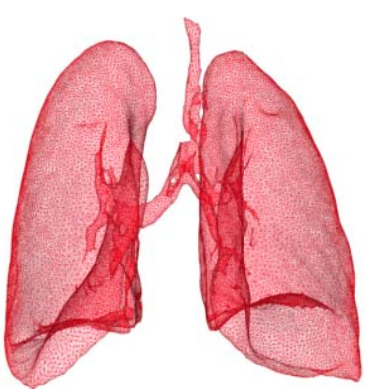

(c) Image 3 (CT3)

Figure 7: Lung CT models.

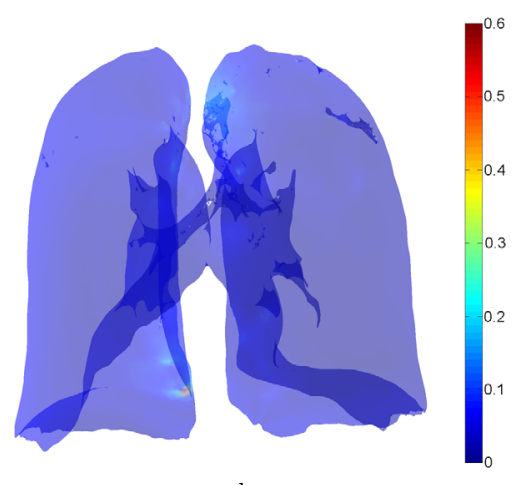

color-map

Figure 8: Color-painting of abnormalities of $C T 1$.

suggests, $C T 2$ has the least degree of abnormalities while the most serious abnormal deformation took place in $C T 4$. To complete the analysis on the deformations, we proceed to visualize the position of abnormal deformations.

As in the previous subsection, we suggest to use color-map to locate the position of large abnormal deformations. To save space we show here only the result of $C T 1, C T 2$, and $C T 3$ (See Figures 8, 9, 10).

Using the images (Figures 8-10), the position of abnormalities can be well approximated for future studies by medical doctors.

\section{Conclusion and future work}

This paper presents an extension of the detection of shape deformities, from that between 2-dimensional surfaces to that between any n-dimensional ob- 


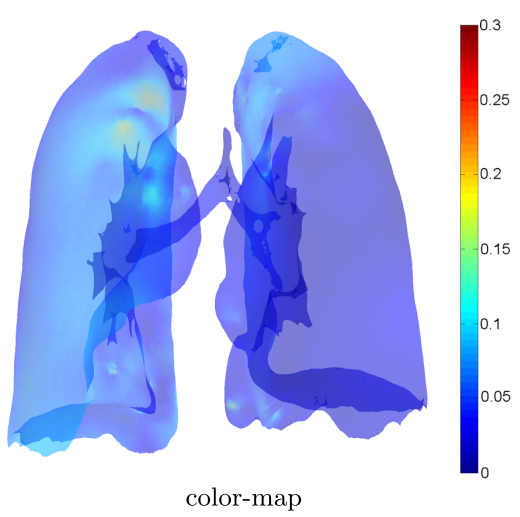

Figure 9: Color-painting of abnormalities of $C T 2$.

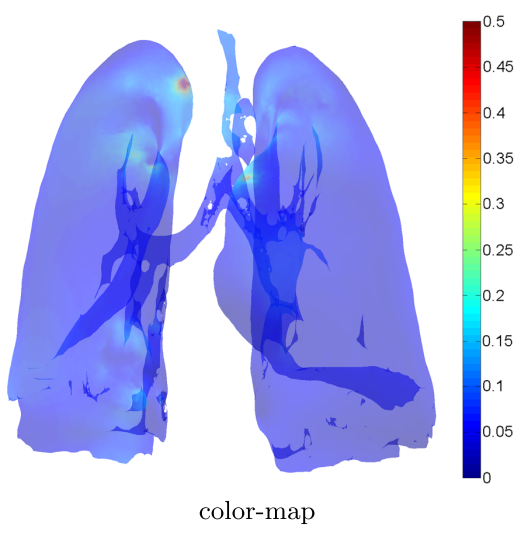

Figure 10: Color-painting of abnormalities of $C T 3$.

jects. By modelling abnormal deformations by anisotropic deformations, we propose the Anisotropic Indicator to quantify the abnormality of a deformation. The basic idea is to characterize a deformation using its local behaviour on an infinitesimal sphere. The proposed indicator provides local information including the position and degree of abnormalities. Also, the indicator is easy to compute in a discrete case. Experimental results approves the effectiveness and sensitivity of our algorithm to detect and extract abnormal deformations. Besides, visualization of abnormalities can be easily achieved using our algorithm, so as to help tracing the regions of abnormalities efficiently. In the future, more experiments on other real medical data will be done to test the algorithm. 


\section{Acknowledgements}

The authors acknowledge the freely available volumetric brain mesh from iso2mesh: a matlab-based 3D tetrahedral mesh generator (http://iso2mesh. sourceforge.net), and the freely available lung CT data from the Deformable Image Registration Laboratory (http://www.dir-lab.com).

\section{References}

[1] D. Tosun, A. L. Reiss, A. D. Lee, R. A. Dutton, K. M. Hayashi, U. Bellugi, A. M. Galaburda, J. R. Korenberg, D. L. Mills, A. W. Toga, and P. M. Thompson. Use of 3-D cortical morphometry for mapping increased cortical gyrification and complexity in Williams Syndrome, IEEE International Symposium on Biomedical Imaging, 1172-1175, 2006.

[2] M. K. Chung, K. J. Worsley, S. Robbins, T. Paus, J. Taylor, J. N. Giedd, J. L. Rapoport, and A. C. Evans. Deformation-based surface morphomtry applied to gray matter deformation, NeuroImage, 18, 198-213, 2003.

[3] Y. Shi, A. L. Reiss, A. D. Lee, R. A. Dutton, U. Bellugi, A. M. Galaburda, J. R. Korenberg, D. L. Mills, I. Dinov, P. M. Thompson, and A. W. Toga. Hamilton-Jacobi skeletons on cortical surfaces with applicatons in characterizing the gyrification pattern in Williams Syndrome, IEEE International Symposium on Biomedical Imaging, 660-663, 2007.

[4] M. Liu, L. Lu, X. Ye, S. Yu, and M. Salganicoff. Sparse classification for computer aided diagnosis using learned dictionaries, Medical Image Computing and Computer-Assisted Intervention (MICCAI) 2011, Springer, Berlin, Heidelberg, 41-48, 2011.

[5] Y. Song, W. Cai, H. Huang, Y. Zhou, D. Feng, and M. Chen. Large margin aggregation of local estimates for medical image classification, Medical Image Computing and Computer-Assisted Intervention (MICCAI) 2014, Springer International Publishing, 196-203, 2014.

[6] H. Kim, B. C. Bernhardt, J. Kulaga-Yoskovitz, B. Caldairou, A. Bernasconi, and N. Bernasconi. Multivariate hippocampal subfield analysis of local MRI intensity and volume: Application to temporal lobe epilepsy, Medical Image Computing and Computer-Assisted Intervention (MICCAI) 2014, Springer International Publishing, 170-178, 2014 . 
[7] L. M. Lui, T. W. Wong, W. Zeng, X. Gu, P. M. Thompson, T. Chan, and S. T. Yau. Detection of shape deformities using Yamabe flow and Beltrami coefficients, Inverse Problem and Imaging, 4(2), 311-333, 2010. MR2653701

[8] Y. Wang, L. Lui, T. Chan, and P. Thompson. Optimization of brain conformal mapping with landmarks, Proceeding in Medical Image Computing and Compute-Assisted Internvention (MICCAI) 2005, 675-683, 2005.

[9] L. Lui, S. Thiruvenkadam, Y. Wang, P. Thompson, and T. Chan. Optimized conformal surface registration with shape-based landmark matching, SIAM Journal of Imaging Sciences, 3(1), 52-78, 2010. MR2609458

[10] L. Lui, Y. Wang, T. Chan, and P. Thompson. Landmark constrained genus zero surface conformal mapping and its application to brain mapping research, Applied Numerical Mathematics, 57, 847-858, 2007. MR2322453

[11] L. Lui, S. Thiruvenkadam, Y. Wang, T. Chan, and P. Thompson. Optimized conformal parameterization of cortical surfaces using shape based matching of landmark curves, Proceeding in Medical Image Computing and Compute-Assisted Internvention (MICCAI) 2005, 494-502, 2008.

[12] F. L. Bookstein. Principal warps: Thin-plate splines and the decomposition of deformations, IEEE Transactions on Pattern Analysis and Machine Intelligence, 11(6), 567-585, 1989.

[13] D. Tosun, M. Rettmann, and J. Prince. Mapping techniques for aligning sluci across multiple brains, Medical Image Analysis, 8, 295-309, 2004.

[14] S. Joshi, and M. Miller. Landmark matching via large deformation diffeomorphisms, IEE Transactions on Image Processing, 26(6), 853-865, 2007.

[15] J. Glaunes, A. Qiu, M. Miller, and L. Younes. Large deformation diffeomorphic metric curve mapping, International Journal of Computer Vision, 80(3), 317-336, 2005.

[16] J. Glaunes, L. Younes and A. Trouve. Landmark matching via large deformation diffeomorphisms on the sphere, Journal of Mathematical Imaging and Vision, 20, 179-200, 2004. MR2049789

[17] J. Glaunes, A. Qiu, M. Miller, and L. Younes. Diffeomorphic matching of distributions: A new approach for unlabelled point-sets and sub- 
manifolds matching, IEEE Computer Society Conference on Computer Vision and Pattern Recognition (CVPR04), 2, 712-718, 2004.

[18] J. Glaunes, A. Qiu, M. Miller, and L. Younes. Surface mathcing via currents, Proceedings of Information Processing in Medical Imaging (IPMI05), Vol. 3565, 381-392, 2005.

[19] W. Zeng, and X. Gu. Registration for 3D surfaces with large deformations using quasi-conformal curvature flow, IEEE Conference on Computer Vision and Pattern Recognition (CVPR11), Jun 20-25, 2011.

[20] Y. T. Lee, K. C. Lam, and L. M. Lui. Large deformation registration via n-dimensional quasi-conformal maps, arXiv preprint, arXiv: 1402.6908, 2014. MR3278842

HEI LONG CHAN

RM 222B, LADY SHAW BUILDING

The Chinese University of Hong Kong

HONG KONG

E-mail address: hlchan@math.cuhk.edu.hk

LOK Ming LuI

RM 207, LADY SHAW BuILDing

The Chinese University of Hong Kong

HONG KONG

E-mail address: lmlui@math.cuhk.edu.hk

ReCEIVEd 17 April 2015 